\title{
Aspecto nutricional do biocarvão na produção de mudas de rúcula em condições semiáridas
}

Joabe Freitas Crispim, Jailma Suerda Silva de Lima, Jessika Kaliane Barbosa da Silva, Antônio Gideilson Correia da Silva, Gabriel Kariel Ferreira Ferdandes, José Elinaldo Alves Bento

Universidade Federal da Paraíba - UFP. Universidade Federal Rural do Semi-Árido - UFERSA. E-mail: crispinjoabef@gmail.com

\section{Resumo}

O biocarvão obtido da pirólise de biomassa vegetal confere à planta um bom suprimento nutricional e possui elevada capacidade de retenção de água, porém ainda há poucos estudos avaliando seu uso na produção de mudas de hortaliças folhosas. Com isso, o objetivo deste trabalho foi determinar proporções de biocarvão em areia que possibilitem melhor desenvolvimento de mudas de rúcula. $O$ experimento foi conduzido em casa de vegetação da Universidade Federal Rural do Semi-Árido (UFERSA). O delineamento experimental utilizado foi o inteiramente casualizado com seis tratamentos $(0 ; 10 ; 20 ; 30 ; 40 ; 50 \%$ de biocarvão) e dez repetições. As características avaliadas foram: Geminação; Número de folhas por planta; Comprimento da raíz; Altura de plantas; Diâmetro do colo; Massa fresca e seca de plantas e teor de água na planta. Os dados foram submetidos à análise de variância. O procedimento de ajustamento de curvas de resposta foi realizado utilizando-se o software R. Recomenda-se a proporção de $50 \%$ de biocarvão na produção de mudas de rúcula por propiciar melhor desenvolvimento nas plantas, promovendo melhorias significativas em todas as características analisadas, exceto germinação e número de folhas por planta.

Palavras-chave: biochar; Eruca sativa; produção de mudas.

\section{Nutritional aspect of biochar in the production of arugula seedlings in semiarid conditions}

\begin{abstract}
The biochar obtained from pyrolysis of vegetable biomass gives the plant a good nutritional supply and has a high water retention capacity, however there are still few studies evaluating its use in the production of leafy vegetable seedlings. With that, the objective of this work was to determine proportions of biochar in sand that allow better development of rocket seedlings. The experiment was conducted in a greenhouse at the Federal Rural University of the Semi-Arid (UFERSA). The experimental design used was completely randomized with six treatments $(0 ; 10 ; 20 ; 30 ; 40 ; 50 \%$ of biochar) and ten replications. The evaluated characteristics were: Twinning; Number of leaves per plant; Root length; Plant height; Neck diameter; Fresh and dry mass of plants and water content in the plant. The data were submitted to analysis of variance. The procedure for adjusting the response curves was performed using the $\mathrm{R}$ software. The $50 \%$ biochar proportion is recommended in the production of arugula seedlings as it provides better development in the plants, promoting significant improvements in all the characteristics analyzed, except germination and number of leaves per plant.
\end{abstract}

Keywords: biochar; Eruca sativa; seedlings production. 


\section{Introdução}

A rúcula (Eruca sativa) é uma hortaliça folhosa de ciclo curto, pertencente à família Brassicaceae, de grande aceitação mundial. No Brasil sua produção e consumo são destacados nas regiões Sul, Sudeste e Nordeste, gerando emprego e renda à agricultura familiar (GRANGEIRO et al., 2011).

A obtenção de mudas vigorosas e bem nutridas é determinante para o estabelecimento da cultura e constitui-se numa das etapas mais importantes do sistema produtivo hortícola. Sendo Assim, para a escolha do substrato na produção de mudas deve-se considerar algumas propriedades inerentes ao mesmo, capazes de fornecer à planta condições ideais para assegurar um bom desenvolvimento inicial.

Assim, a característica do substrato utilizado é de fundamental importância na definição do potencial produtivo da planta. Isso ocorre porque, na produção de mudas dentro de um recipiente, o crescimento do sistema radicular e a absorção de nutrientes ficam restritos ao volume do substrato contido no interior do recipiente utilizado. Nesse contexto, o substrato ideal deve ter baixa densidade, alta capacidade de troca catiônica, alta capacidade de retenção de água e boa aeração e drenagem, proporcionando condições ideais para o crescimento e desenvolvimento de mudas (TERRA et al., 2011).

O sistema de produção de mudas tem enfrentado outros problemas relacionados à dependência de insumos químicos dispendiosos no modelo convencional, traduzindo-se em desvantagens para o sistema. Os fertilizantes químicos, e o impacto ambiental negativo gerado pelo manejo inadequado dos mesmos têm atingindo também a qualidade dos alimentos produzidos (BATISTA et al., 2016), fortalecendo assim a tendência de uso de sistemas totalmente orgânicos.

Dessa forma, o biocarvão, também conhecido como "Biochar", atende adequadamente aos requisitos ideais necessários à produção de mudas de hortaliças. Esse composto é resultante da pirólise de materiais orgânicos de origem animal, vegetal, da agroindústria ou até mesmo de culturas voltadas à sua produção. Em sua composição possui alto teor de nutrientes e grande área superficial devido à porosidade (BENITES et al., 2005). Vale ressaltar ainda que o fato de o biocarvão ser produzido a partir de qualquer fonte de biomassa contribui para sua disponibilidade, facilitando sua aquisição.

Os efeitos benéficos do biochar são determinados principalmente por algumas de suas propriedades: alta porosidade, responsável por sua alta capacidade de retenção de água; alta capacidade de troca catiônica, o que favorece a retenção de nutrientes e evita sua perda; fornecimento direto de nutrientes e a capacidade de ser habitat de microrganismos benéficos, que podem promover a liberação e a absorção de nutrientes pelas plantas (ATKINSON et al., 2010)

Souchie et al. (2011) em estudo com carvoeiro (Tachigali paniculata Aubl.), observaram que o substrato com biochar influenciou a taxa de germinação de sementes, apresentando boa qualidade como condicionador. Silva et al. (2019) verificaram que a adição de biochar, produzido a partir serragem de eucalipto e adicionado ao substrato comercial 'Bioplant ${ }^{\circledR}$ ', na concentração de 5,0 \%, apresentou viabilidade para ser utilizado como condicionador de substrato, possuindo ainda baixo custo se comparado com os condicionantes comerciais.

Diante do exposto, o objetivo deste trabalho é avaliar a resposta de mudas de rúcula a diferentes proporções de biocarvão como condicionador de substrato em areia lavada.

\section{Material e Métodos}

O experimento foi conduzido em casa de vegetação na Universidade Federal Rural do Semi-Árido (UFERSA), localizada no município de Mossoró-RN, no período de 16 de janeiro a 09 de fevereiro de 2018.

O delineamento experimental utilizado foi o inteiramente casualizado com seis tratamentos e quatro repetições. Os tratamentos consistiram de diferentes proporções de biocarvão em areia lavada $(0 ; 10 ; 20 ; 30 ; 40 ; 50 \%$ de biocarvão). Cada repetição foi composta por dez plantas. As proporções foram calculadas e dadas em porcentagem com base no peso de 120 gramas do substrato total, desse modo, o último tratamento, por exemplo, foi composto por $60 \mathrm{~g}$ de biocarvão e $60 \mathrm{~g}$ de areia. O biocarvão utilizado foi produzido a partir da biomassa de espécies vegetais nativas e abundantes do semiárido. A semeadura foi realizada em copos plásticos de $150 \mathrm{~mL}$ previamente furados para evitar o acúmulo prejudicial de água. Após uma semana da semeadura foi realizado o desbaste, deixando-se apenas uma planta por copo. Foi 
utilizada a variedade 'Cultivada', com um ciclo de 30 a 40 dias.

Os copos foram mantidos em casa de vegetação. Durante a condução do experimento foram realizadas duas irrigações diárias (manhã e tarde) com o uso de um regador. A água foi proveniente de um poço da universidade (tabela 1).

Tabela 1. Resultados da análise da água do poço da horta experimental da UFERSA, Mossoró-RN.

\begin{tabular}{|c|c|c|c|c|c|c|c|c|c|c|c|c|}
\hline $\mathrm{CE}$ & $\mathrm{pH}$ & $\mathrm{K}$ & $\mathrm{Na}$ & $\mathrm{Ca}$ & $\mathrm{Mg}$ & $\mathrm{HCO}_{3}$ & $\mathrm{CO}_{3}$ & $\mathrm{Cl}$ & RAS & Dureza & Cátions & Ânions \\
\hline $\mathrm{dS} / \mathrm{m}$ & & & & & $--m$ & olc/L--- &  & & & $\mathrm{mg} / \mathrm{L}$ & ----mm & c/L---- \\
\hline 0,556 & 8,3 & 0,24 & 3,79 & 0,8 & 0,3 & 0,2 & 2,0 & 2,0 & 5,1 & 55 & 5,1 & 4,2 \\
\hline
\end{tabular}

As avaliações foram realizadas 24 dias após a semeadura. As características avaliadas foram: Germinação; Número de folhas por planta; Altura da planta e comprimento da raiz, determinados através de uma régua graduada; Diâmetro do colo, determinado com o auxílio de um paquímetro digital; Massa fresca de plântulas, obtida através da pesagem de cinco plantas completas em balança analítica; Massa seca de plântulas, obtida através da secagem das cinco plantas anteriores, as quais foram colocadas em

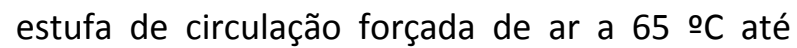
atingir massa constante; teor de água na planta, obtido da diferença entre massa fresca e seca.

Os dados foram submetidos à análise de variância pelo aplicativo software SAS. As análises de regressão foram feitas pelo software $R$.

\section{Resultados e Discussão}

Houve efeitos significativos da elevação das proporções de biocarvão para todas as características, exceto germinação e número de folhas por planta.

Obteve-se uma média de $92 \%$ no percentual germinativo das sementes da rúcula, não havendo, praticamente, alteração na germinação conforme elevação nas proporções do biocarvão. Esse resultado é excelente, e o fato de não haver efeito da elevação dessas proporções se deve à qualidade da semente associada à potencialidade da areia para germinação (DUARTE et al., 2019; BRASIL, 2009), fazendo com que as proporções complementares do condicionador de substrato não tivessem influência significativa sobre a característica. Esse resultado também indica que o biocarvão produzido a partir de plantas da caatinga não possui compostos tóxicos capazes de inibir a germinação de sementes de rúcula.

Obteve-se um número médio de 3,5 folhas por planta, não havendo resposta ao aumento das proporções do biocarvão. provavelmente o curto tempo de avaliação não foi suficiente para demonstrar um possível efeito do fator nessa característica. Desse modo, para se averiguar adequadamente as respostas do número de folhas ao aumento do biocarvão seria necessário levar o trabalho a campo e fazer a contagem de novas brotações.

Houve aumento no comprimento de raízes e na altura de plantas à medida que se elevou as proporções de biocarvão, obtendo-se um valor máximo de 3,32 e $5,5 \mathrm{~cm}$, respectivamente, na maior dose complementar de $50 \%$ (figura $1 \mathrm{~A}$ e $\mathrm{B}$ ). Esse ganho pode ser explicado pelo aumento do $C$ orgânico total e do $\mathrm{N}$ total causado por maiores proporções do biocarvão (SCHULZ et al., 2013). Schulz et al (2014) estudando o efeito do biocarvão no crescimento das plantas em estufa, verificaram que quantidades baixas desse material não tem ação significativa sobre o crescimento vegetal por causa da baixa disponibilidade de nutrientes. 
Figura 1. Comprimento de raízes (A), altura de plantas (B), diâmetro do colo (C), massa fresca (D), massa seca (E) e teor de água (F) de mudas de rúcula em função de proporções de biocarvão em areia lavada.
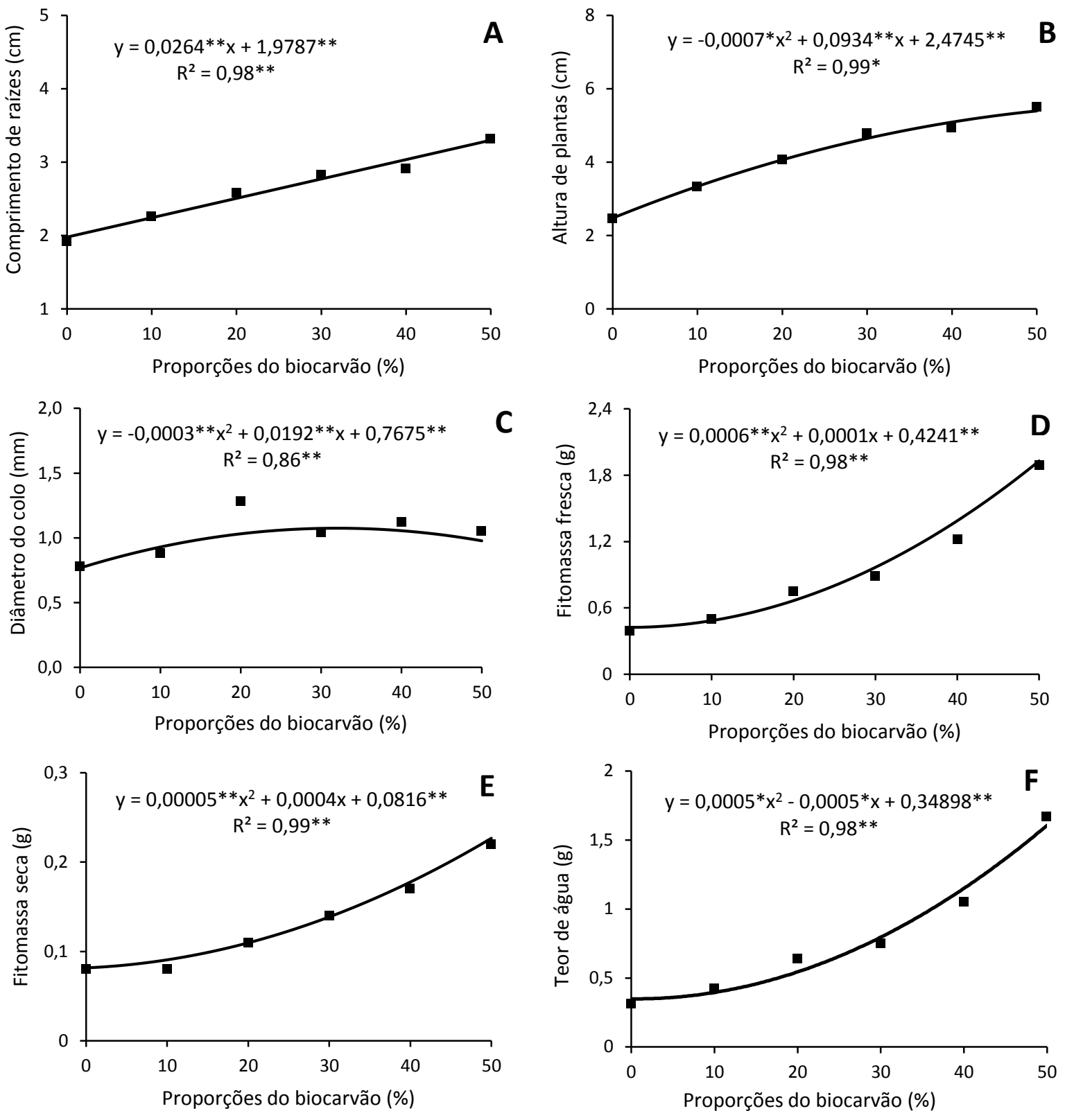

Ao se comparar a presente pesquisa com os resultados observados na literatura, verifica-se que o incremento nas variáveis observadas está sempre correlacionado de forma positiva com a elevação das proporções do biocarvão, havendo melhores resultados sempre nas maiores proporções (JAISWAL et al., 2015; SCHULZ et al., 2013; CAVALCANTE et al., 2012). Entretanto a quantidade ótima pode variar com a cultura trabalhada e ainda com a composição do substrato. Geralmente, os efeitos do biocarvão no crescimento das plantas e nas propriedades físico-químicas dos meios de cultivo variam de acordo com a matéria-prima do substrato, o tamanho das partículas e o processo de

produção, por exemplo, temperatura e duração do aquecimento (BELDA et al., 2016; CHO et al., 2017; GEORGE et al., 2017). Dessa forma, o tipo de material de origem é fator determinante na indução de respostas da planta.

No diâmetro do colo, verifica-se que houve um comportamento crescente até a proporção de $20 \%$ de biocarvão, onde se observou um valor de $1,28 \mathrm{~mm}$, decrescendo em seguida e mantendo-se praticamente estável até a última proporção aplicada do biocarvão (Figura 1C). O diâmetro do colo é um dos melhores parâmetros indicadores de padrão de qualidade, estando relacionado com a segurança e estabilidade da planta em campo. As mudas de 
pequeno diâmetro e muito altas são consideradas de qualidade inferior quanto àquelas de maior diâmetro de colo. Ademais, um maior diâmetro de colo está associado a um desenvolvimento mais acentuado da parte aérea e, em especial, do sistema radicular, favorecendo a sobrevivência e o desenvolvimento da muda após o plantio (GRAVE et al., 2007).

Para massa fresca e seca de plantas observou-se comportamento crescente em função das doses complementares, obtendo-se 1,89 e $0,22 \mathrm{~g}$, respectivamente, na maior proporção de biocarvão de 50\% (Figura 1D e E). Esse desempenho deve ser explicado pelo aumento dos benefícios físicos e químicos do biocarvão, em função das doses crescentes do mesmo, refletindo positivamente no investimento da planta em fitomassa. Novotny et al. (2009) confirmam que o biocarvão possui em sua estrutura molecular sítios capazes de realizar troca iônica, condição singular que pode contribuir para o aumento da capacidade de troca catiônica (CTC) dos substratos e assim proporcionar maior disponibilidade de nutrientes. $O$ teor de água nas plantas também aumentou significativamente com a elevação nas proporções do biocarvão, saindo de 0,31 até 1,67 g na última quantidade estudada (figura $1 \mathrm{~F}$ ). $\mathrm{O}$ incremento observado nessas características também está relacionado aos benefícios do biocarvão como condicionador de atributos físicos capazes de promover no substrato maior porosidade, permitindo, dessa forma, melhor desenvolvimento da muda, bem como maior retenção de água.

\section{Conclusão}

O aumento nas proporções de biochar em areia lavada influencia positivamente a produção de mudas de rúcula.

Recomenda-se o uso do biocarvão como condicionador de substrato na proporção de $50 \%$, por melhorar significativamente as características que compõem a qualidade das mudas de rúcula.

Em futuros trabalhos, torna-se pertinente estudar maiores proporções do biocarvão, averiguando a influência desse fator não só com as características aqui avaliadas, mas também na firmeza do torrão e na taxa de pegamento das mudas em campo.

\section{Referências}

ATKINSON, C. J.; FITZGERALD, J. D.; HIPPS, N. A. Potential mechanisms for achieving agricultural benefits from biochar application to temperate soils: a review. Plant and soil, v. 337, n. 1-2, p. 118, 2010. https://DOI 10.1007/s11104-010-0464$\underline{5}$

BATISTA, M. A.; BEZERRA NETO, F.; SILVA, M. L.; AMBRÓSIO, M. M.; CUNHA, J. L. Atributos de solo-planta e de produção de beterraba influenciados pela adubação com espécies da Caatinga. Horticultura Brasileira, v. 34, n. 1, p. 31-38, 2016. https://doi.org/10.1590/S0102$\underline{053620160000100005}$

BELDA, R. M.; LIDÓN, A.; FORNES, F. Biochars and hydrochars as substrate constituents for soilless growth of myrtle and mastic. Industrial Crops and Products, v. 94, p. 132-142, 2016.

https://doi.org/10.1016/j.indcrop.2016.08.024

BENITES, V. M.; MENDONÇA, E. S.; SCHAEFER, C. E. G. R.; NOVOTNY, E. H.; REIS, E. L.; KER, J. C. Properties of black soil humic acids from high altitude rock complexes in Brazil. Geoderma v. 127, n. 1-2, p. 104-113, 2005.

https://doi.org/10.1016/j.geoderma.2004.11.020

BRASIL. Ministério da Agricultura e Reforma Agrária. Regras para análise de sementes. Brasília: Mapa/ACS, 2009. 399 p.

CAVALCANTE, I. H. L.; PETTER, F. A.; ALBANO, F. G.; SILVA, R. R. S.; SILVA JÚNIOR, G. B. Biochar no substrato para produção de mudas de maracujazeiro amarelo. Revista de la Facultad de Agronomía, La Plata, v. 111, n. 1, p. 41-47, 2012.

CHO, M. S.; MENG, L.; SONG, J. H.; HAN, S. H.; $B A E, K$.; PARK, B. B. The effects of biochars on the growth of Zelkova serrata seedlings in a containerized seedling production system. Forest science and technology, v. 13, n. 1, p. 25-30, 2017.

https://doi.org/10.1080/21580103.2017.1287778

DUARTE, M. M.; FREITAS MILANI, J. E.; OLIVEIRA, T. W. G.; BLUM, C. T.; NOGUEIRA, A. C. Seed morphobiometry and germination of Sesbania punicea (cav.) benth. and Sesbania virgata (cav.) pers. Floresta, v. 49, n. 4, p. 661-670, 2019. http://dx.doi.org/10.5380/rf.v49i4.57410

GEORGE, E.; VENTURA, M.; PANZACCHI, P.; SCANDELLARI, F.; TONON, G. Can hydrochar and pyrochar affect nitrogen uptake and biomass 
allocation in poplars? Journal of Plant Nutrition and Soil Science, v. 180, n. 2, p. 178-186, 2017. https://doi.org/10.1002/ipln.201600563

GRANGEIRO, L. C.; OLIVEIRA, F.; NEGREIROS, M.; MARROCOS, S.; LUCENA, R.; OLIVEIRA, R. Crescimento e acúmulo de nutrientes em coentro e rúcula. Revista Brasileira de Ciências Agrárias, v. 6, n. 1, p. 11-16, 2011.

https://doi.org/10.5039/agraria.v6i1a634

GRAVE, F.; FRANCO, E. T. H.; PACHECO, J. P.; SANTOS, S. R. crescimento de plantas jovens de açoita-cavalo em quatro diferentes substratos. Ciência Florestal, v. 17, n. 4, p. 289-298, 2007. https://doi.org/10.5902/198050981961

JAISWAL, A. K.; FRENKEL, O.; ELAD, Y.; LEW, B.; GRABER, E. R. Non-monotonic influence of biochar dose on bean seedling growth and susceptibility to Rhizoctonia solani: the "Shifted R max-Effect". Plant and soil, v. 395, n. 1-2, p. 125140, 2015. https://DOI 10.1007/s11104-014$\underline{2331-2}$

NOVOTNY, E. H.; HAYES, M. H.; MADARI, B. E.; BONAGAMBA, T. J.; AZEVEDO, E. R. D.; SOUZA, A. A. D.; MANGRICH, A. S. Lessons from the Terra Preta de Índios of the Amazon region for the utilisation of charcoal for soil amendment. Journal of the Brazilian Chemical Society, v. 20, n. 6, p. 1003-1010, 2009.

https://doi.org/10.1590/S0103-

$\underline{50532009000600002}$

SCHULZ, H.; DUNST, G.; GLASER, B. No effect level of co-composted biochar on plant growth and soil properties in a greenhouse experiment. Agronomy, v. 4, n. 1, p. 34-51, 2014. https://doi.org/10.3390/agronomy4010034

SCHULZ, H.; DUNST, G.; GLASER, B. Positive effects of composted biochar on plant growth and soil fertility. Agronomy for Sustainable Development, v. 33, n. 4, p. 817-827, 2013. https://doi.org/10.1007/s13593-013-0150-0

SILVA, L. F. V.; MELO, E. I.; GONÇALVES, P. A. S. Biochar de serragem de eucalipto como condicionador de substratos para produção de mudas de alface. Agri-environmental sciences, $v$. 5, e019005,

2019. https://doi.org/10.36725/agries.v5i0.1614
SOUCHIE, F. F.; MARIMON JUNIOR, B. H.; PETTER, F. A.; MADARI, B. E.; MARIMON, B. S.; LENZA, E. Carvão pirogênico como condicionante para substrato de mudas de Tachigali vulgaris L.G. Ciência Florestal, v. 21, n. 4, p. 811-821, 2011. http://dx.doi.org/10.5902/198050984526

TERRA, S. B.; FERREIRA, A. A. F.; PEIL, R. M. N.; STUMPF, E. R. T.; BECKMANN CAVALCANTE, M. Z.; CAVALCANTE, I. H. L. Alternative substrates for growth and production of Potted chrysanthemum (cv. Funny). Acta Scientiarum. Agronomy, v. 33, n. 3, p. 465-471, 2011.

https://doi.org/10.4025/actasciagron.v33i3.6991 